\title{
EL ENFOQUE LÚDICO COMO DIDÁCTICA PARA FACILITAR LA COMUNICACIÓN ASERTIVA
}

\author{
García Ruth $^{1}$, Andrea Pozo ${ }^{2}$, Erika Casa ${ }^{3}$,Patricia Anangono ${ }^{4}$ \\ ruth0277@hotmail.com ${ }^{1}$, elizandy-ep@hotmail.com²,ms.educacion81inicial@gmail.com ${ }^{3}$, \\ patygps8@gmail.com4 \\ https://orcid.org/0000-0002-0044-2488 ${ }^{1}$ https://orcid.org/0000-0003-0037-6975 \\ https://orcid.org/0000-0001-7562-1678 ${ }^{3}$, \\ Ministerio de Educación1 2 3-Universidad Nacional de Rosario ${ }^{123}$, Universidad de Salamanca ${ }^{4}$ \\ Quito-Ecuador
}

Recibido (03/08/20), Aceptado (14/08/20)

\begin{abstract}
Resumen: La influencia del presente artículo radica en comprobar lo beneficioso que es para una comunicación asertiva el contar con un nuevo léxico logrado mediante el enfoque lúdico. Para ello se llevó a cabo la aplicación de estrategias encaminadas a la adquisición de un nuevo vocabulario que será conducente en la dotación de herramientas comunicativas. Por tal razón el estudio expuesto justifica su valía en el enfoque teórico vygotskyano sobre la virtud del juego, beneficios prácticos educativos y notabilidad social con la importancia de la comunicación humana. Todo esto se desarrolló bajo el paradigma cuantitativo de tipo descriptivo, cuya muestra responde a un tipo no probabilístico conformada por 80 estudiantes de octavo EGB, empleando como instrumento la evaluación objetiva de tipo diagnóstico. Derivando en un significativo cuadro comparativo de calificaciones como la consecuencia del antes y después de la aplicación de la propuesta, dando notoriedad a la apropiación de un nuevo vocabulario en la capacidad comunicativa.
\end{abstract}

Palabras Clave: Comunicación humana, lúdica, estrategias léxicas.

\section{PLAYFUL APPROACH AS A DIDACTIC TO FACILITATE ASSERTIVE COMMUNICATION}

\begin{abstract}
The influence of this article lies in verifying how beneficial it is for an assertive communication to have a new lexicon achieved through the playful approach. For this, the application of strategies aimed at acquiring a new vocabulary was carried out, which will be consistent in the acquisition of communication tools. For this reason the exposed study justifies its value in the Vygotskyan theoretical approach about the virtue of the game, practical educational benefits and social notability with the importance of human communication. The development of this research is focused on the quantitative paradigm and it is descriptive, whose sample responds to a non-probabilistic type made up of eighty students of eighth grade of Basic General Education, using objective diagnostic-type evaluation as an instrument. Deriving in a significant comparative table of qualifications, as the consequence of before and after the application of the proposal, giving notoriety to the appropriation of a new vocabulary in the communicative capacity.
\end{abstract}

Keywords: Human communication, playful, lexical strategies. 


\section{I.INTRODUCCIÓN}

El léxico es definido como el conjunto de palabras de un idioma, recibe también el nombre de vocabulario [1] . La importancia del aprendizaje de un nuevo léxico radica en que la falta de dominio activo de vocabulario receptivo o productivo ocasiona malos entendidos, inseguridades, limitaciones y bloqueo en la comunicación. Si se conoce a la palabra así como a su definición, el aprendizaje se vuelve motivante y dinámico permitiendo fortalecer las habilidades lingüísticas que a su vez potencializan la comprensión dialéctica y facilitan la comunicación cotidiana tan indispensable en la convivencia social en todas las áreas y tiempos [2].

La comunicación asertiva por si sola ya es compleja y el aprendizaje de esta habilidad se torna aún más, debiendo tener en cuenta que el éxito de esta práctica radica en trasmitir y recibir los mensajes, sentimientos, creencias u opiniones propios o ajenos de una manera honesta, oportuna y respetuosa [3]. En este contexto, se puede mencionar la necesidad de fortalecer estrategias léxicas con enfoque lúdico implementadas de manera didáctica en la formación académica de los estudiantes en todos los niveles y subniveles de la educación formal.

En tal virtud la lúdica es más utilizada como técnica educativa que ayuda al estudiante a desarrollar la creatividad, interés, con el fin de potencializar sus capacidades para la solución de problemas, es decir, que al aplicar esta estrategia, a más de adquirir conocimiento, desarrolla la capacidad creativa del educando, fortaleciendo relaciones comunicativas idóneas [2]. La significancia de la lúdica en el aula depende del docente y del interés que despierte en los estudiantes, para que de esa forma, cree un clima propicio donde la dinámica se el conductor del conocimiento, permitiendo el desarrollo de la creatividad y renovación en los educandos, para poder integrar y poner en práctica el nuevo léxico adquirido.

Con el propósito de puntualizar la problemática en educación y de modo preciso en el área de Lengua y comunicación es necesario clarificar que en la actualidad no se responde a las exigencias que demanda la sociedad, corroborándolo con los datos estadísticos proporcionados por el Instituto Nacional de Evaluación [4]. El informe de resultados del Tercer estudio regional comparativo y explicativo (Terce) de la Organización de las Naciones Unidas para la Educación la Ciencia y la Cultura aplicada a estudiantes de 4to, 7mo, 10mo año de básica y 3ro de bachillerato, que reflejan porcentajes mínimos [5]. Otro instrumento que también asevera dicha información es la prueba diagnóstica dirigida específicamente a los estudiantes de séptimo año. Por lo tanto, los resultados de las tres evaluaciones presentan porcentajes mínimos en las destrezas léxicas.

Este trabajo investigativo tiene como objetivo resaltar la importancia de la adquisición de un nuevo léxico sustentado en el aspecto lúdico como factor de aprendizaje, coadyuvando de esta manera a mejorar los niveles y prácticas comunicativas en los educandos. Además, desarrollando competencias profesionales al plantear la lúdica como una alternativa didáctica-pedagógica que contribuya al mejoramiento del proceso de aprendizaje en general. Permitiendo así mismo socializar las experiencias obtenidas mediante la investigación de campo cuantitativa y para la interpretación de datos el proceso deductivo secuencial.

El presente artículo está organizado en cuatro secciones, la primera expone la conceptualización e importancia de la temática a tratar, en la segunda el desarrollo de la problematización, la tercera presenta el sustento investigativo y finalmente en la cuarta sección se conjugan los objetivos con la metodología.

\section{II.DESARROLLO}

\section{A.La lengua oral y la comunicación oral}

Es así que, considerando que se habla para aprender, surge lo que los autores denominan la Metodología Conversacional, que propone que los objetivos de aprendizaje de la lengua oral sean considerados eje transversal en todas las asignaturas escolares, y que el proceso de enseñanza-aprendizaje considere el discurso oral como objeto y mediador, de modo que la comunicación oral fluya en el aula y con las comprensiones [6]. La percepción, el medio, la memorización y el razonamiento son acciones concretas que ha de desarrollar el individuo frente a esa capacidad de dar lenguaje a las imágenes tangibles de las cosas surgidas, una vez que se inicia la dinámica misma de la comunicación propiamente dicha [7] .

Otros autores defienden que el lenguaje en el contexto escolar es y debe ser considerado una "herramienta de comunicación e instrumento mediador en los procesos de enseñanza y aprendizaje" [6]. En tal virtud se puede afirmar que el conocimiento disposicional obedece a las emociones e imágenes, los mismos que pueden ser innatos o adquiridos acorde a las experiencias sensitivas mediatizadas por la comunicación del individuo [7].

\section{B.El léxico como elemento fundamental para una comunicación asertiva}

Son muchos los factores que inciden en una correcta o inapropiada comunicación, siendo determinante la comprensión, el cociente intelectual, la fluidez lectora 
y el vocabulario intrínsecamente relacionados en todo este proceso. Otros estudios relacionan esta problemática (comunicación asertiva) con variables cognitivas y procesos de naturaleza meta cognitiva de la persona [8].

Como punto destacado de lo antes señalado podemos aclarar que la adquisición de vocabulario proporciona una base importante para el desarrollo del lenguaje y que es básico en prácticas de alfabetización, incluida la comprensión de lectura y la identificación de palabras. En este sentido, los niños deben tener un repertorio de vocabulario adecuado para poder comprender el texto. Asimismo, cuando los niños pueden usar claves contextuales que apoyan la identificación de palabras, así como desarrollan habilidades de alfabetización como ortografía, conciencia fonológica y semántica simultáneamente, lo que les permite identificar y comprender palabras. [8].

\section{C.Niveles de adquisición en el desarrollo léxico y su valía}

El vocabulario o léxico para llegar a su consolidación en la adquisición del lenguaje debe atravesar por varias etapas: a) Desarrollo fonológico: Esta etapa va de 0 a 4 años, corresponde a la actividad fónica que sirve de base para el funcionamiento de los órganos para concretar el lenguaje, de esta forma predispone al niño para el proceso de captación. b) Desarrollo léxico: El proceso que se presenta en el niño es paulatino, empezando por nombres de objetos para después emplear palabras como sustantivos, adjetivos, verbos y de esa forma ir enriqueciendo su léxico. En este sentido, pueden presentarse errores léxicos como sobreextención que significa que el niño emplea un mismo término para referirse a diferentes elementos y por otro lado la subextensión que consiste en utilizar un término general para referirse a un tema definido. c) Desarrollo gramatical Etapa comprendida entre los 9 y 18 meses, el estilo o etapa holofrástica empleada señala que el niño fusiona varias palabras en un solo valor comunicacional. d) Desarrollo discursivo Esta fase tardía hace que el niño aprenda, emplee conectores y estos le permitan entrelazar ideas y palabras para el acto comunicativo [2].

En estos últimos años, el estudio del léxico se ha tornado de vital importancia, sobre todo para los estudiantes de educación básica, es así, muchos docentes de la carrera de Lengua española, han realizado sus trabajos finales de maestría en esta temática, el objetivo ha sido, buscar estrategias, metodologías y actividades para lograr que los estudiantes a partir de su léxico usual, incorporen un léxico disponible, el mismo que permitirá emplear nueva terminología en determinados contextos [2]. Por otro lado, diversas organizaciones locales como internacionales han realizado estudios sobre esta temática con la finalidad de identificar si la problemática radica en los estudiantes, maestros, técnicas, metodología, didáctica, currículo u otros factores. Los resultados de estos trabajos demuestran una falencia proporcional en varios componentes, siendo la didáctica un elemento iterativo mayoritariamente.

\section{D.Enfoque lúdico didáctico}

Con este antecedente contextualizado en el campo educativo y ligado inherentemente al orden social comunitario, es imprescindible priorizar principios didácticos que en el campo pedagógico viabilicen alternativas de solución prácticas, justificadas desde el campo del saber. En este sentido, la lúdica es utilizada como una técnica educativa que ayuda al estudiante a desarrollar la creatividad, interés, con el fin de potencializar sus capacidades para la solución de problemas, es decir, que al aplicar esta estrategia, a más de adquirir conocimiento, desarrollará la capacidad creativa del educando, también ayudará a establecer relaciones comunicativas interpersonales [2]. Por ello destaca Pellis en [9], jugando es como preparamos nuestro cerebro para la vida, el amor e incluso el trabajo de aprendizaje.

Los juegos deben considerarse como una actividad importante en el aula, puesto que aportan una forma diferente de adquirir el aprendizaje mediante el descanso y la recreación. Los juegos permiten orientar el interés del participante hacia las áreas que se involucren en la actividad lúdica. El profesor/a hábil y con iniciativa, inventa juegos que se acoplen a los intereses, las necesidades, las expectativas, la edad y el ritmo de aprendizaje [9]. Con todo esto la lúdica puede y de hecho debe ser utilizada para el proceso de enseñanza aprendizaje en la adquisición de vocabulario, de tal manera que se constituya en un vehículo para adquirir destrezas lingüísticas, El valor didáctico de la lúdica depende del docente, $\mathrm{y}$ del interés que despierte en los alumnos, para que de esa forma, cree un clima propicio donde la dinámica sea el conductor del conocimiento, permitiendo el desarrollo de la creatividad y renovación en los educandos, para poder integrar el nuevo léxico adquirido [2].

\section{III.METODOLOGÍA}

La población objeto de estudio responde a una muestra de tipo no probabilístico conformada por 80 estudiantes de octavo año de educación básica de la institución educativa Consejo Provincial de sostenimiento fiscal ubicada en la ciudad de Quito, integrada por 58 niñas $(72.5 \%)$ y 22 niños $(27.5 \%)$, cuyas edades fluctúan entre los 11 y 12 años de edad.

El instrumento aplicado fue la evaluación objetiva 
de tipo diagnóstico, encaminado a detectar debilidades y fortalezas en el ámbito lingüístico, basado en destrezas con criterio de desempeño e inherentes al bloque de escritura y comunicación oral del referente curricular emitido por el Ministerio de Educación, es así que los conocimientos lingüísticos (léxico, semántica, sintáctica y fonología) serán medidos en la decodificación y compresión de textos. La evaluación consta de tres reactivos, estos son: repuesta breve, ordenamiento y emparejamiento, está estructurado de 6 ítems, cada uno con temática diferente. El primer ítem trato la inferencia de significados, el segundo valoró hiperónimos e hipónimos, de igual manera el tercer ítem tiempos verbales, el cuarto atendió sinónimos, el quinto midió la estructura y cohesión de la oración simple y finalmente el sexto agregado, palabras variables. Para el análisis del presente trabajo se empleó el método de cálculo estadístico descriptivo e inferencial.

Los estudiantes que participaron en esta labor lo hicieron de forma voluntaria y con la colaboración de docentes del área de lengua y literatura en la jornada escolar regular, con los respectivos permisos de los órganos regulares que viabilizan este tipo de trabajos.

\section{IV.RESULTADOS}

Los resultados obtenidos en la evaluación diagnóstica muestran de manera general que el nivel del léxico en este grupo de estudiantes es insuficiente, sin embargo, en el reactivo relacionado a la cohesión y coherencia al ordenar oraciones el porcentaje es alto, denotando que en esta destreza no hay mayor grado de dificultad.
Los efectos extraídos en este trabajo no distan de los expuestos por [4] y el [5] puesto que corroboran el planteamiento mostrando porcentajes mínimos que van ligados al bajo nivel de dominio en esta área objeto de análisis. Se deduce también que es necesario implementar actividades didácticas que fortalezcan el léxico de los estudiantes mejorando de esta manera la calidad de comunicación y por ende los niveles académicos.

Con lo que respecta a la propuesta; se planificó una serie de actividades que permitan la adquisición de un nuevo vocabulario, mismo que conjugaría actividades lúdicas en su didáctica. Estas actividades fueron desarrolladas en períodos consecutivos de clase, cabe señalar que los estudiantes en esta institución educativa reciben 6 horas de clase a la semana y cada hora clase o pedagógica cuenta con 40 minutos, por lo tanto, se designó 3 horas semanales para su ejecución. Los resultados de las acciones a corto plazo se evidenciaron durante y después de cada clase, observando mayor predisposición e interés en la asignatura [2].

$\mathrm{Su}$ implementación en la práctica de actividades léxicas sustento su accionar en competencias comunicacionales más relevantes, mismas que parten de fundamentos teóricos abordados en el trabajo. Tal es así que se enfatiza en el tema del lenguaje y su adquisición, la lúdica como factor para el aprendizaje, una comunicación asertiva aplicando un nuevo vocabulario, entre otros.

A continuación, se registran algunas tablas de actividades como referencia de la propuesta ejecutada.

TABLA I. Actividades inherentes a la adquisición de vocabulario sesión número 1.

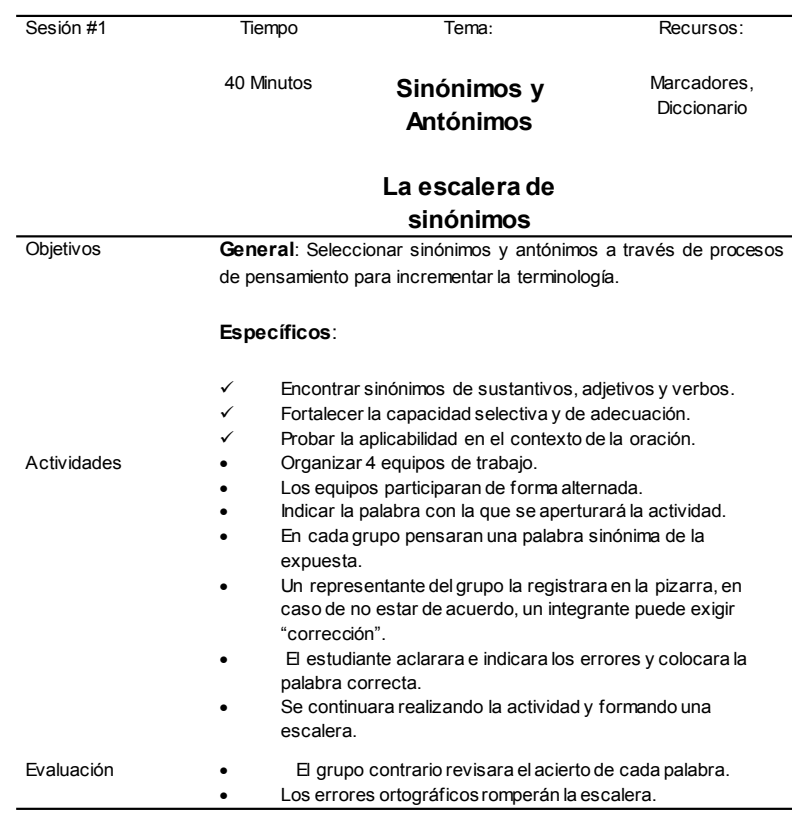

Fuente: unidad educativa Consejo Provincial. Elaborado por las autoras 
TABLA II. Actividades inherentes a la adquisición de vocabulario sesión número 3.

\begin{tabular}{|c|c|c|c|}
\hline Sesión \#3 & $\begin{array}{c}\text { Tiempo } \\
40 \\
\text { Minutos }\end{array}$ & $\begin{array}{c}\text { Tema: } \\
\text { Super Híper }\end{array}$ & $\begin{array}{c}\text { Recursos: } \\
\text { Hojas-Esferos }\end{array}$ \\
\hline \multirow[t]{2}{*}{ Objetivos } & \multicolumn{3}{|c|}{$\begin{array}{l}\text { General: Escribir palabras hipónimas a partir del hiperónimo dado } \\
\text { para ampliar su léxico. }\end{array}$} \\
\hline & $\begin{array}{ll}\checkmark & \text { Desa } \\
\checkmark & \text { Busc } \\
\checkmark & \text { Forta } \\
\checkmark & \text { Regis }\end{array}$ & $\begin{array}{l}\text { creatividad } \\
\text { nos de los hiperónim } \\
\text { apacidad selectiva y } \\
\text { ayor número de pala }\end{array}$ & ación. \\
\hline Actividades & $\begin{array}{ll}\text { - } & \text { Forn } \\
\text { - } & \text { Inves } \\
& \text { pres } \\
\text { - } & \text { Recc } \\
& \text { palal } \\
\text { - } & \text { Real } \\
\text { - } & \text { Inser }\end{array}$ & $\begin{array}{l}\text { s de } 5 \text { estudiantes } \\
\text { evas palabras de } \\
\text { grupo que tiene e } \\
\text { imer borrador del } \\
\text { alabras investigad }\end{array}$ & húmero de \\
\hline Evaluación & Le & nto & \\
\hline
\end{tabular}

Fuente: unidad educativa Consejo Provincial. Elaborado por las autoras

TABLA III. Actividades inherentes a la adquisición de vocabulario sesión número 5.

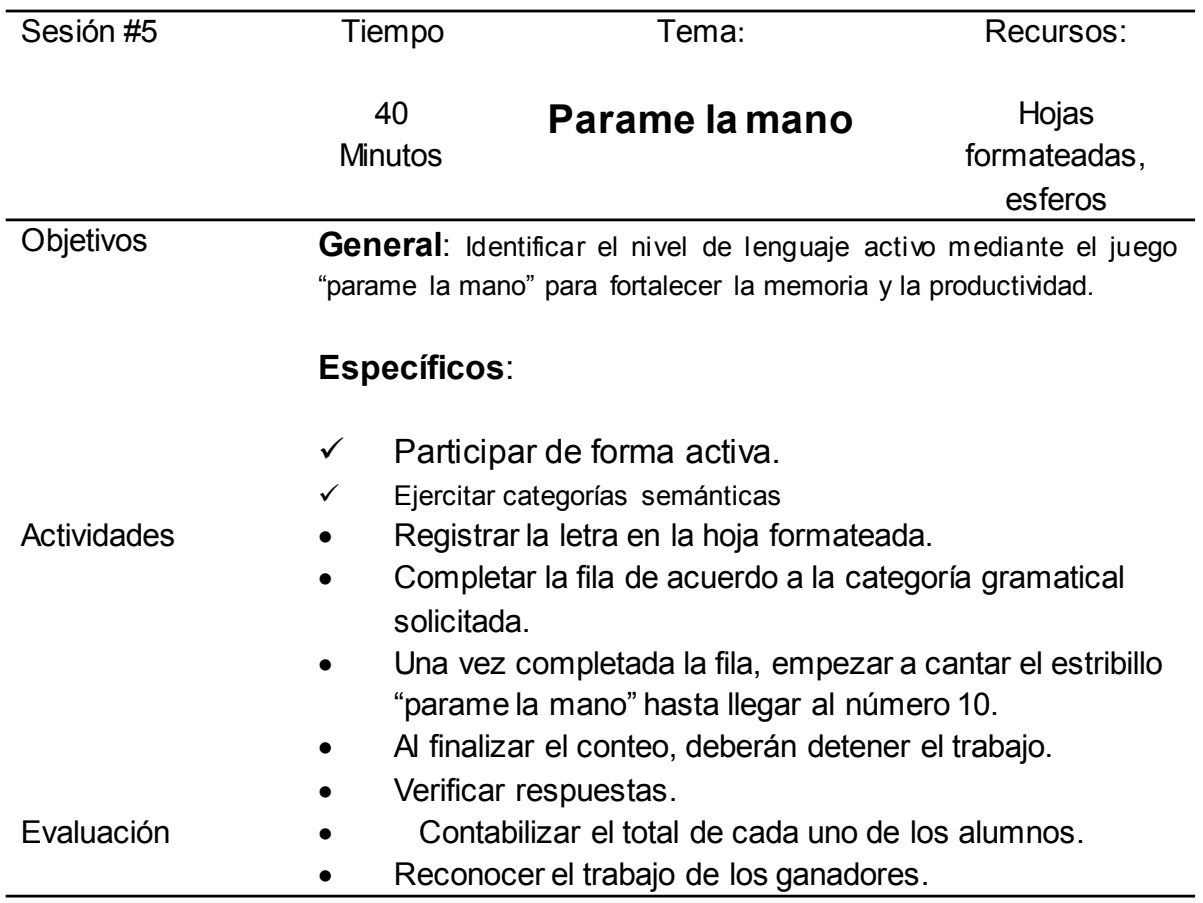

Fuente: unidad educativa Consejo Provincial. Elaborado por las autoras

En cuanto a las actividades expuestas, podemos observar el nivel de planificación e incorporación de la lúdica como herramienta didáctica para el aprendizaje de un nuevo léxico y que los resultados obtenidos de esta aplicación reflejen un avance significativo en el rendimiento académico de los estudiantes en todas las áreas, por lo tanto, la propuesta se convirtió en una herramienta eficaz para mejorar destrezas propuesta pero además se evidencia una mejora académica considerable en todas las asignaturas. Es un hecho que la buena comprensión comunicativa optimiza el proceso de aprendizaje.

A continuación, se presenta una tabla comparativa 
con el avance cuantitativo que se evidenció posterior a la implementación de las actividades propuestas.

TABLA IV. Cuadro de rendimiento académico.

NOMBRE DEL PROFESOR

PATRICIA ANANGONO

Materia

Lengua y Literatura

Nómina
RTE S (3-5)

QUINTO PARCIAL

SEXTO PARCIAL

\begin{tabular}{|c|c|c|c|c|c|c|c|c|c|c|c|c|}
\hline $\begin{array}{c}\text { Materia } \\
\text { Lengua y Literatura } \\
\text { Nómina }\end{array}$ & 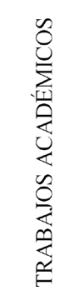 & 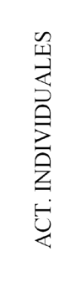 & 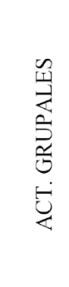 & 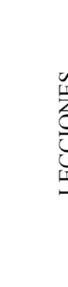 & 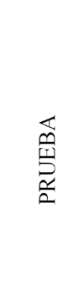 & 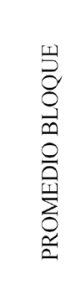 & 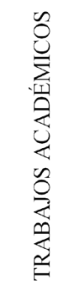 & 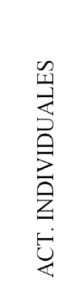 & 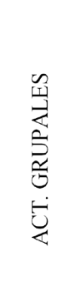 & 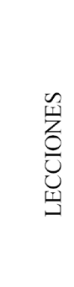 & 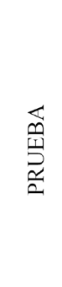 & $\begin{array}{l}5 \\
8 \\
0 \\
0 \\
0 \\
0 \\
0\end{array}$ \\
\hline iA MEDRANO ANDRES SEBASTIAN & 1,67 & 7,83 & 8,50 & 6,03 & 8,00 & 6,40 & 7,00 & 5,00 & 8,25 & 7,00 & 10,0 & 7,45 \\
\hline VAREZ ALARCON BRIGITTE DANIELA & 3,33 & 7,90 & 8,70 & 6,80 & 7,50 & 6,80 & 9,00 & 8,00 & 8,25 & 7,50 & 10,0 & 8,55 \\
\hline ZAMORA COSTA MAYERLY DALAY & 9,57 & 9,57 & 9,00 & 8,80 & 8,90 & 9,20 & 10,0 & 9,60 & 9,25 & 10,0 & 7,22 & 9,21 \\
\hline MIJO HIDALGO JOSELIN CAROLINA & 8,37 & 7,87 & 7,80 & 6,20 & 8,70 & 7,80 & 9,10 & 8,20 & 9,50 & 10,0 & 10,0 & 9,35 \\
\hline RAHONA REGALADO DENISSE ELA & 6,60 & 9,07 & 8,20 & 7,00 & 9,70 & 8,10 & 9,66 & 9,90 & 8,00 & 7,50 & 5,55 & 8,12 \\
\hline JAMARCA TIGSE JHOSELIN ABIGAIL & 6,00 & 5,00 & 4,00 & 7,00 & 5,00 & 5,40 & 7,00 & 8,00 & 9,00 & 7,00 & 8,00 & 7,80 \\
\hline MACHO JIMENEZ MELANIE VALERIA & 9,77 & 9,67 & 9,40 & 8,10 & 9,00 & 9,20 & 9,93 & 9,90 & 9,75 & 8,00 & 9,00 & 9,31 \\
\hline MPOS PASUY SARAHI ELIZABETH & 8,00 & 5,60 & 8,70 & 5,40 & 7,80 & 7,10 & 7,83 & 8,50 & 8,25 & 10,0 & 10,0 & 8,91 \\
\hline NDO QUILLUPANGUI MELANY PAOLA & 9,93 & 9,33 & 8,90 & 7,40 & 10,00 & 9,10 & 10,0 & 9,90 & 9,50 & 7,50 & 7,22 & 8,82 \\
\hline IQUIN LICERO EVELIN PAULINA & 1,00 & 8,00 & 8,40 & 5,70 & 9,00 & 6,40 & 9,50 & 8,30 & 7,75 & 9,00 & 5,00 & 7,91 \\
\hline AZ CHUQUIMARCA MAYERLY STEFY & 8,23 & 8,27 & 8,40 & 6,60 & 6,60 & 7,60 & 9,26 & 8,60 & 8,75 & 10,0 & 8,00 & 8,92 \\
\hline RZON MERINO ALISSON MELISSA & 4,00 & 9,07 & 8,70 & 7,00 & 7,00 & 7,10 & 6,33 & 6,30 & 8,00 & 9,80 & 7,22 & 7,52 \\
\hline IILLEN ARTEAGA JORDAN DAVID & 2,00 & 9,80 & 9,50 & 8,20 & 5,00 & 6,90 & 9,90 & 9,90 & 9,50 & 10,0 & 10,0 & 9,16 \\
\hline IISCASO TOAPANTA ADRIANA ALISSA & 6,93 & 9,00 & 9,20 & 7,20 & 6,00 & 7,70 & 9,93 & 9,90 & 8,50 & 7,50 & 6,11 & 8,83 \\
\hline IACHAMIN ROSERO DAYANA RIGETT & 9,77 & 8,43 & 8,90 & 9,00 & 7,60 & 8,70 & 9,87 & 9,90 & 8,40 & 9,80 & 6,60 & 8,28 \\
\hline ARRA CHICAIZA MILEIDY MARGARITA & 5,93 & 7,67 & 7,90 & 6,40 & 7,00 & 7,00 & 9,00 & 8,50 & 7,50 & 9,80 & 6,60 & 8,28 \\
\hline ZANO PALADINES LAURA STEFANY & 7,00 & 9,60 & 9,70 & 8,20 & 7,00 & 8,30 & 9,93 & 10,0 & 10,0 & 10,0 & 7,77 & 9,53 \\
\hline :DINA ARMIJOS SHASALY RENATA & 5,00 & 8,50 & 8,80 & 6,30 & 8,70 & 7,40 & 8,93 & 9,40 & 7,50 & 10,0 & 10,0 & 9,16 \\
\hline INA TITUAÑA PAOLA ALEXANDRA & 6,00 & 9,60 & 6,00 & 8,70 & 6,90 & 7,40 & 10,0 & 10,0 & 9,40 & 8,00 & 9,44 & 9,36 \\
\hline
\end{tabular}

Fuente: unidad educativa Consejo Provincial. Elaborado por las autoras

Posterior a lo observado en el cuadro que nos antecede, no cabe duda que otra teoría que avala este trabajo es la consecuencia de la comunicación humana, pues se afirma que toda conducta es comunicación, y toda comunicación afecta a la conducta. Atañe el efecto que de la comunicación sobre el receptor y el efecto que la reacción del receptor tiene sobre el emisor [10].

\section{V.CONCLUSIONES}

Los resultados obtenidos en la aplicación de las actividades propuestas, reflejan un avance significativo en el rendimiento académico de los estudiantes en todas 
las áreas, por lo tanto, la misma se ha convertido en una herramienta eficaz para mejorar competencias tanto lingüísticas (comunicación) como en otros campos (social). En el cuadro de rendimiento académico. Tabla IV. Podemos evidenciar la variación en lo que respecta a las notas antes y depués de la ejecución del plan, ante ello consentimos sintetizar que las estrategias léxicas empleadas en actividades didácticas lúdicas fortalecieron los aspectos inherentes a optimar.

El llevar a la práctica la implementación de actividades léxicas que fortalezcan las debilidades detectadas en los estudiantes, ha distinguido metas comunicacionales jerárquicamente preponderantes que parten de fundamentos teóricos abordados en el estudio. Por tal razón se debe enfatizar en el accionar práctico del área de lenguaje: aportes pedagógicos, psicológicos, la adquisición de un nuevo léxico, la lúdica como factor didáctico, la comunicación humana, entre otros. Cabe recalcar que toda actividad intencionalmente planificada en el orden académico científico aporta en el contexto global del ser humano como tal, así lo demuestra el presente artículo que vinculó destrezas de aprendizaje lingüísticos con habilidades de convivencia social.

La culminación de la propuesta ha permitido que tanto docentes y estudiantes potencialicen el trabajo en cada hora pedagógica, así mismo el poseer un vasto vocabulario mejoró notablemente los niveles de comprensión y por ende una comunicación en feedback. Posterior a este estudio se han vivenciado experiencias comunicativas satisfactorias influyendo en el rendimiento académico, así como también a una visible mejora en su comportamiento comunicacional colectivo. Por tal virtud la comunicación humana intensifica las relaciones sociales que a su vez caracterizan un óptimo rendimiento en cualquier labor del ser humano encomendada, premisa que nos permitirá abrir canales de investigación futuros por la repercusión global y fáctica que le atañe.

\section{REFERENCIAS}

[1]RAE, «Real Academia Española,» Octubre 2014. [En línea]. Available: https://dle.rae.es/1\%C3\%A9xico. [Último acceso: 05 Julio 2020].
[2]A. G.Patricia, mplementación de actividades léxicas en estudiantes de octavoaño de educación básicaa través de estrategias lúdicas., Quito, Pichincha, 2018.

[3]R. Martín Vegas, «AUGE E PIPDAL,» 2017. [En línea].Available: http://epipdal.auge.edu.es/epipdal/pluginfile.php/44841/mod resource/content/12/ USAL_LEN_LIT_A10_T02.pdf. [Último acceso: JULIO 2017].

[4] INEVAL, «Informe de resultados Ser Bachiller ciclo 2014-2015,» Publicaciones Ineval, Quito, 2017.

[5]UNESCO, «Informe de resultados TERCE: logros de aprendizaje,» Organización de las Naciones Unidas para la Educación, la Ciencia y la Cultura, Francia, 2016.

[6]L. Hermida y M. Grácia, «USO DE LA APLICACIÓN DIGITAL EVALOE-SSD COMO INSTRUMENTO DE DESARROLLO DOCENTE PARA MEJORAR LAS COMPETENCIAS COMUNICATIVAS Y LINGÜÍSTICAS DE LOS ALUMNOS DE EDUCACIÓN INICIAL,» UNIVERSIDAD CIENCIA Y TECNOLOGÍA, p. 49, 2019.

[7]J. Paguay Balladares, D. Carpio Vera y G. Logroño Valdez, "APROXIMACIÓN TEÓRICA DE LA ACCIÓN COMUNICATIVA Y SU RELACIÓN CON LA NEUROCIENCIA COGNITIVA,» UNIVERSIDAD CIENCIA Y TECNOLOGÍA, p. 140, 2019.

[8]J. Anilema Guamán, R. Moreta Herrera y M. Mayorga Lascano, «DIAGNÓSTICO DE LA COMPRENSIÓN LECTORA EN ESTUDIANTES DEL CANTÓN COLTA, ECUADOR,» UNIVERSIDAD CIENCIA Y TECNOLOGÍA, p. 65, 2020.

[9]J. G. López Araujo, A. E. Pozo Potosí, Y. C. Bodero Aguayo y N. J. Loor Aguayo, «EL JUEGO EN EL DESARROLLO INTELECTUAL DEL NIÑO,» UNIVERSIDAD CIENCIA Y TECNOLOGÍA, pp. 97-106, 2020.

[10]V. Rojas y R. Calle, «PROGRAMACIÓN NEUROLINGÜÍSTICA EN LA GENERACIÓN DE REDES AFECTIVAS PARA LA RESOLUCIÓN DE CONFLICTOS EN ESPACIOS PEDAGÓGICOS,» UNIVERSIDAD CIENCIA Y TECNOLOGÍA, pp. 5-10, 2019. 


\section{RESUMEN CURRICULAR}

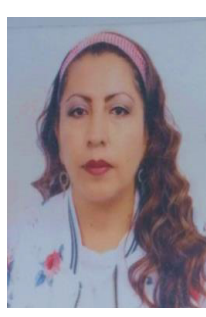

García, Ruth; Magister en Pedagogía de nacionalidad ecuatoriana, en la actualidad directivo de una institución educativa emblemática del sistema educativo fiscal gracias a su conocimiento y título en licenciatura con mención en gerencia y liderazgo educativo, amante de la lectura e investigación de las neurociencias y procesos educativos, razón por la cual su título inaugural lo obtuvo en el instituto superior Normal Manuela Cañizares con alusión en docencia de educación básica. En este momento se encuentra cursando el Doctorado en Ciencias de la Educación en la Universidad Nacional de Rosario - Argentina.

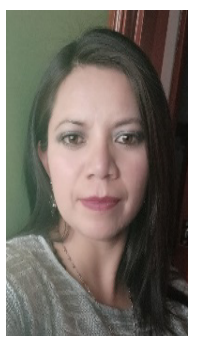

Pozo Potosi, Andrea Elizabeth; Magister en Educación y Proyectos de Desarrollo con Enfoque de Género, Licenciada en Ciencias de la Educación mención Profesora Parvularia, de nacionalidad ecuatoriana, docente del nivel Inicial en el Ministerio de Educación, apasionada de la lectura y la investigación. Cursando el Doctorado en Ciencias de la Educación en la Universidad Nacional de Rosario - Argentina.

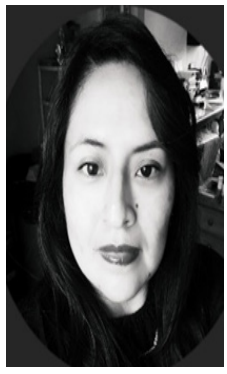

Casa Llano Erika Silvia; Magister en Educación Inicial, Licenciada en Ciencias de la Educación mención Profesora Parvularia, nacionalidad ecuatoriana, en la actualidad directivo de un Centro de Desarrollo Para la Primera Infancia Ministerio de Educación, su amor por la enseñanza en la educación Inicial, le ha motivado día a día para buscar siempre cambios e innovación en su área. Actualmente se encuentra cursando un Doctorado en Ciencias de la Educación en la Universidad Nacional de Rosario -Argentina. https:/casa-erika.com/ 